OPEN ACCESS

Edited by:

Lydia Sam,

University of Aberdeen,

United Kingdom

Reviewed by:

Helen Mavromichalaki,

National and Kapodistrian University of

Athens, Greece

Jingnan Guo,

University of Science and Technology

of China, China

Krzysztof Iskra,

Military University of Aviation, Poland

*Correspondence:

Patrícia Gonçalves

patricia@lip.pt

Specialty section:

This article was submitted to

Planetary Science,

a section of the journal

Frontiers in Astronomy and Space

Sciences

Received: 10 December 2021

Accepted: 26 January 2022

Published: 21 February 2022

Citation:

Goncalves $P$, Arruda $L$ and Pinto $M$ (2022) Validation of dMEREM, the Detailed Mars Energetic Radiation Environment Model, with RAD Data

from the Surface of Mars.

Front. Astron. Space Sci. 9:833144.

doi: 10.3389/fspas.2022.833144

\section{Validation of dMEREM, the Detailed Mars Energetic Radiation Environment Model, with RAD Data from the Surface of Mars}

\author{
Patrícia Gonçalves ${ }^{1,2 *}$, Luísa Arruda $^{2}$ and Marco Pinto ${ }^{2}$ \\ ${ }^{1}$ Departamento de Física e Departamento de Engenharia e Ciências Nucleares, Instituto Superior Técnico, Universidade de \\ Lisboa, Lisboa, Portugal, ' ${ }^{2}$ IP- Laboratório de Instrumentação e Física Experimental de Partículas, Lisboa, Portugal
}

The detailed Martian Energetic Radiation Environment Model (dMEREM) is a Geant4 based model developed for the European Space Agency, which enables to predict the radiation environment expected at different locations on the Martian orbit, atmosphere and surface, as a function of epoch, latitude and longitude, taking into account the specific atmospheric and soil composition, based on different particle propagation codes for primary Galactic Cosmic Rays or Solar Particle Events. This work describes the validation of dMEREM with differential proton fluxes measured with the NASA Curiosity rover Radiation Assessment Detector (RAD) at the Gale Crater, at the surface of Mars, from 15 November 2015 to 15 January 2016 and in the beginning of September 2017, using two different Galactic Cosmic Ray (GCR) models, the ISO-15390 and the Badhwar-O'Neill 2014 models. This work includes a comparative study of the available Geant4 physics lists in describing the RAD measured proton spectrum, and an investigation of the proton directional spectrum at the Martian surface, and on its effect on the comparisons between models and data, which are necessarily measured within a limited field-of-view. For both GCR models and data periods there was a good agreement between the proton fluxes in the energy range between 10 and $90 \mathrm{MeV}$ measured at the surface of Mars with RAD and the corresponding dMEREM predictions. Therefore, although the RAD only measures a limited field-of-view in zenith angle of the Martian Particle Radiation Field, and this effect has to be taken into account, the results obtained constitute an important benchmark in the use of dMEREM in the assessment of the expected ionising radiation field on the surface of Mars.

Keywords: mars radiation environment, Geant4 (G4), RAD (radiation assessment detector), GCR, proton differential fluxes

\section{INTRODUCTION}

The characterization of the Martian radiation environment is of major importance for Mars exploration. Radiation levels expected at the surface of Mars are known to have an impact on life forms, biological, organic materials (Baumstark-Khan and Facius, 2001), (Cucinotta, 2012), (Cucinotta et al., 2010), (Durante and Cucinotta, 2008) and semiconductor devices, emphasising the need for well trusted and validated transport codes. The radiation environment on Mars differs greatly from Earth's mainly due to Mars not having a global magnetic field, only weak crustal magnetic fields, and also because of the sparse Martian atmosphere, with column depths of 15-22 g/ 
$\mathrm{cm}^{2}$ at the surface, 50 to 70 to times lower than the $1,030 \mathrm{~g} / \mathrm{cm}^{2}$ average atmospheric depth on Earth at sea level. Both facts contribute to a weak shielding against energetic particles at the Martian surface, resulting not only on higher levels of radiation at Mars when compared to Earth, but also on a very different composition of the radiation fields. It is therefore crucial to study the Martian radiation environment and the way it affects spacecraft systems and crews. This is especially relevant to evaluate the health risks for astronauts in future Mars missions and to establish radiation hazard mitigation strategies.

The radiation field at the Martian surface is mainly induced by Galactic Cosmic Rays (GCR), which interact in the sparse Martian atmosphere and with the soil materials producing secondary particles. GCR are mainly composed by protons (90\%), helium nuclei (9\%), electrons (1\%) and fully ionized heavy nuclei (Simpson, 1983). Their energies range from values lower than $1 \mathrm{MeV} /$ nuc up to $\sim 1 \mathrm{EeV} /$ nuc, with maximum intensities around a few $\mathrm{GeV}$, following negative power-law distributions for higher energies (Nymmik et al., 1992; O'Neill, 2010). The fluxes of GCR decrease below the $\mathrm{GeV} /$ nuc region due modulation by the solar activity, displaying higher average energies, but a lower fluences, during solar maximum periods than during solar minimum periods (Adams ea, 1981). GCR penetrate the sparse Martian atmosphere originating secondary particles that propagate in particle showers. Additionally, GCR protons with energy above $\sim 150 \mathrm{MeV}$ (for atmospheric depths of $\sim 20 \mathrm{~g} / \mathrm{cm}^{2}$ ) (Guo et al., 2018) reach directly the Martian surface and interact with the soil materials originating secondary particles via spallation and fragmentation processes, resulting on an albedo neutrons and energetic gammas, which contribute to the radiation field. The resulting spectrum at the surface of Mars is therefore a combination of different particle types within a broad energy range.

Although GCR constitute the dominant contribution to the radiation environment on Mars, Solar Energetic Particle events (SEPs) - mostly protons and electrons plus 10\% helium nuclei and $<1 \%$ heavier elements -, with energies ranging from a few $\mathrm{keV}$ up to $\sim 1 \mathrm{GeV}$ may be the dominant component of the primary particles arriving on Mars for limited time periods of several days. Comparisons of dMEREM predictions with SEP data collected at the surface of Mars will be reported in a dedicated publication following the present dMEREM validation analysis.

Particle radiation data from the surface of Mars were obtained for the first time with the RAD, the Radiation Assessment Detector (Hassler et al., 2012), an instrument of the NASA Curiosity Rover payload (Grotzinger et al., 2012), in Gale Crater (Cabrol et al., 1999) since August 2012. These first insitu measurements provide an important data set for assessing the radiation-associated risks for future human Mars exploration and valuable inputs for the design of protective habitats and for countermeasures for future missions. RAD was designed to measure differential particle fluxes for lower-energy charged particles: protons, deuterons, tritons, other isotopes of helium, carbon, nitrogen, oxygen and iron ions (up to $\sim 100 \mathrm{MeV} / \mathrm{nuc}$ ) and integral fluxes for higher energies of these ions (Ehresmann et al., 2017). RAD measurements offer a unique opportunity to validate particle transport codes developed to model the Martian radiation environment, with real data from Mars.

Different transport codes like Particle and Heavy Ion Transport Code System (PHITS) (Sato et al., 2013; FloresMcLaughlin, 2017), the High charge ( $Z$ ) and Energy TRaNsport (HZETRN) (Slaba et al., 2016), (Slaba and Stoffle, 2017), Monte Carlo N-Particle (MCNP6) (Ratliff et al., 2017), High Energy Transport Code-Human Exploration and Development in Space (HETC-HEDS) (de Wet and Townsend, 2017) and GEometry ANd Tracking (GEANT4) (Agostinelli et al., 2003; Allison et al., 2006; Allison et al., 2016) are available for studying the particle spectra and radiation dose at Mars (Hassler et al., 2017). In particular, models based on Geant4, such as a PLANETOCOSMICS (Matthiä and Berger, 2017), which simulates particle transport in planetary magnetic fields and atmospheres, and AtRIS, the Atmospheric Radiation Interaction Simulator (Guo et al., 2019), are used to simulate the Martian radiation environment and were already validated with RAD data.

The Mars Energetic Radiation Environment Models, MEREM (Gonçalves et al., 2009), are ionising radiation environment characterization models for Mars, developed for the European Space Agency under the MarsREM project. The detailed Martian Energetic Radiation Environment Model (dMEREM) is a Geant4 based model that predicts the particle radiation environment on Mars atmosphere surface and on Phobos and Deimos (McKennaLawlor et al., 2012a), (McKenna-Lawlor et al., 2012b), (Da Pieve et al., 2021). eMEREM, the engineering Martian Energetic Radiation Environment Model uses a set of pre-computed response functions based on the FLUKA radiation transport code (Ferrari et al., 2005; Böhlen et al., 2014) to calculate the shielding effects of the atmosphere and surface for a range of mono-energetic proton and helium nuclei sources. While dMEREM and eMEREM are each capable of operating in stand-alone mode, they are interfaced with SPENVIS (Heynderickx et al., 2004; Kruglanski et al., 2009), the Space ENVironment Information System web-based user interface, where results of the models' predictions can be extracted in the form of pre-defined csv files. The SPENVIS interface also enables the extraction of input parameters for stand-alone operation of both models. In this paper, dMEREM's predictions validation with data from the Martian surface are described, and AMEREM is shown to be capable of providing a detailed characterization of the different contributions for the radiation environment at the surface of Mars, including spectral and directional information.

\section{MATERIALS AND METHODS}

The detailed Martian Energetic Radiation Environment Model is described in detail in (Gonçalves et al., 2009; McKenna-Lawlor et al., 2012b). Since its release, dMEREM was continuously updated to comply with the current versions of Geant4. In this study Geant4-10.1.2 version was used. The model has also been improved in terms of computing performance and adapted 
for more flexibility of input formats and for more complete output information.

\section{Definition of the Physical Environment in dMEREM}

dMEREM relies on a pre-processor that extracts Martian atmospheric composition and density profiles from data bases and models-from the European Mars Climate Data Base (Forget et al., 1999; Millour et al., 2018) and from MarsGRAM (Justus et al., 2002)- and it uses the data from the Mars Odyssey Gamma Ray Spectrometer (GRS) (Boynton et al., 2004) for the determination of surface soil composition and density. The retrieval of this information is performed as a function of the Martian latitude and longitude, in a $5^{\circ} \times 5^{\circ}$ latitude-longitude grid, and, for the case of the atmospheric data, also as a function of solar longitude and for day and night conditions.

The dMEREM default atmosphere is $50 \mathrm{~km}$ high, and it is divided in 20 layers of similar depth (in $\mathrm{g} / \mathrm{cm}^{2}$ ). A $50 \mathrm{~km}$ high atmosphere corresponds approximately to $99 \%$ of the total atmospheric depth. If optionally crustal magnetic fields are considered, an additional layer with a height of $100 \mathrm{~km}$ is placed on top of the default atmosphere to account for the variability on the trajectories of the particles propagating along the magnetic field lines. The atmosphere description in the data base can be accessed for the column of 20 equal depth atmospheric layers (in $\mathrm{g} / \mathrm{cm}^{2}$ ), for 12 different solar longitude intervals (12 Martian "months"), and for day and night conditions. The parameters describing each atmospheric layer are the average atmospheric density $\left(\mathrm{g} / \mathrm{cm}^{3}\right)$, temperature $(\mathrm{K})$, and weight percentages of $\mathrm{CO}, \mathrm{CO}_{2}, \mathrm{H}_{2}, \mathrm{H}_{2} \mathrm{O}$ ice, $\mathrm{H}_{2} \mathrm{O}$ vapour and $\mathrm{O}_{2}$. Information on the surface pressure $(\mathrm{Pa})$, temperature $(\mathrm{K})$ and weight percentage of $\mathrm{CO}_{2}$ ice at surface are also extracted and stored.

In the case of soil composition a default basalt/andesitic-basalt composition is used, augmented by information on the surface iron, silicon and hydrogen concentration extracted from the GRS Mars Odyssey data. The soil density is then calculated according to the weight percentage of each material.

The simulated 3D dMEREM pixel has then a $50 \mathrm{~km}$ high atmosphere on top of a $3 \mathrm{~m}$ thick soil layer with constant composition and density, which is dependent on the Martian latitude and longitude. The square transverse section of the pixel has default values of $500, \times, 500 \mathrm{~km}^{2}$, which are changed to $900, \times$, $900 \mathrm{~km}^{2}$ if the magnetic field is turned on.

\section{dMEREM Output}

The present stand-alone dMEREM version output adds a ROOT $^{1}$ tree structure to the standard csv pre-defined outputs, which contain integral particle flux information for a chosen sets of particle species and radiological quantities, such as Effective Dose (ED) and Ambient Dose Equivalent (ADE), ED being a protection quantity, corresponding to the sum of dose equivalents in all tissues and organs of the body,

${ }^{1}$ https://root.cern.ch/
TABLE 1 | Basaltic soil composition from APXS analysis of Rocknest soil at Gale Crater (Blake et al., 2013; Hassler et al., 2014).

\begin{tabular}{lcl}
$\begin{array}{l}\text { Gale } \\
\text { Crater soil composition }\end{array}$ & Weight percentage (\%) & Soil density \\
\hline $\mathrm{H}_{2} \mathrm{O}$ & 5.2 & \\
$\mathrm{Fe}_{2} \mathrm{O}_{3}$ & 20.1 & 2.8 \\
$\mathrm{SiO}_{2}$ & 44.9 & $\mathrm{~g} / \mathrm{cm}^{3}$ \\
$\mathrm{Bulk}\left(\mathrm{Al}_{2} \mathrm{MgCaNa}_{2} \mathrm{~K}_{2} \mathrm{O}_{7}\right)$ & 29.8 & \\
\hline
\end{tabular}

and $\mathrm{ADE}$ being the appropriate operational quantity for region monitoring of strongly penetrating radiation, as discussed in (McKenna-Lawlor et al., 2012b). These quantities are calculated using fluence to $\mathrm{ED}$ and fluence to $\mathrm{ADE}$ conversion factors of (Pelliccioni, 2000). The ROOT output structure enables to save information of the characteristics of all individual particles reaching a "sensitive" detector, which is the scoring volume, such as particle species, energy and direction, enabling to perform detailed analyses, verifications and inter-comparisons at post-processing level with additional flexibility.

\section{Particle Scoring}

Primary particles are generated at the centre of the $500 \mathrm{~km} \times$ $500 \mathrm{~km}$ dMEREM pixel, from the top of the simulated atmosphere, at the altitude of $50 \mathrm{~km}$, according to a cosinelaw distribution. All particles resulting from the interactions of primary particles with the atmosphere and soil materials, arriving at a scoring volume covering the $500 \mathrm{~km} \times 500 \mathrm{~km}$ dMEREM pixel at the Martian surface are then tallied and their characteristics stored in the ROOT dMEREM output.

\section{dMEREM Inputs for Validation With RAD Data}

Predictions of radiological quantities with the MarsREM were published in (McKenna-Lawlor et al., 2012b) and found to be in reasonable agreement with those of transport code HZTERN (De Angelis et al., 2007), although these comparisons are indicative, since dMEREM outputs Ambient Dose Equivalent, and HZTERN predictions are given in dose equivalent. For three different characterised sites, the GCR induced ADE rates predicted by dMEREM (and eMEREM) ranged between $0.48 \mathrm{mSv} /$ day for dMEREM at Mawrth Vallis site, and $0.71 \mathrm{mSv} /$ day for dMEREM at Viking landing site, the latter compared to the $0.8 \mathrm{mSv} /$ day dose equivalent rate prediction of HZTERN for similar solar minimum conditions (De Angelis et al., 2007). These values are compatible with the average for the GCR dose equivalent rate on the Mars surface of $0.64 \pm 0.12 \mathrm{mSv} /$ day, measured with the RAD (Hassler et al., 2014). In the present study two different RAD data sets consisting of stopping proton differential fluxes measured at the Gale Crater from 15 November 2015 to 15 January 2016 (Ehresmann et al., 2017) and in the beginning of September 2017 (Ehresmann et al., 2018) are compared to the corresponding AMEREM predictions for the same epochs and location on the Martian surface. 


\section{Nov15-15Jan16}

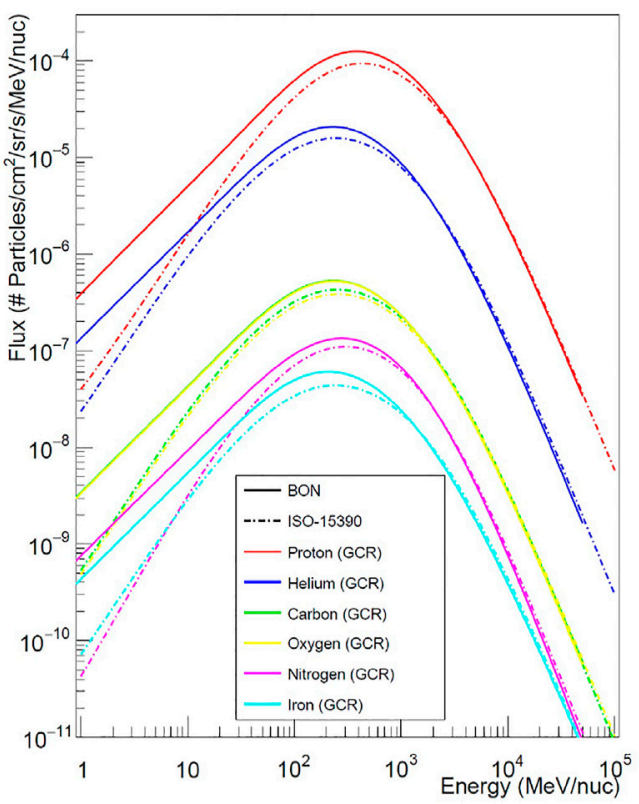

10Sep17

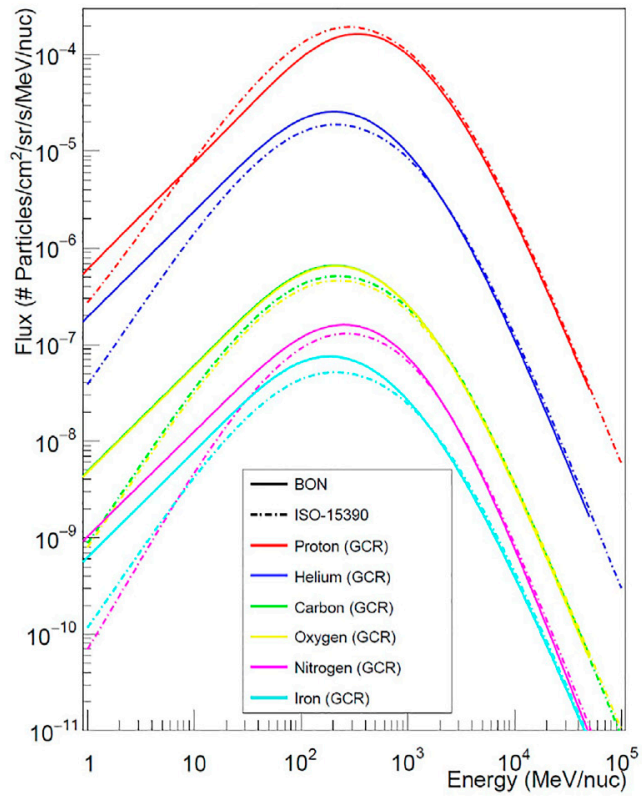

FIGURE 1 | Primary simulated spectra for GCR hydrogen $(Z=1)$, helium $(Z=2)$, carbon $(Z=6)$, nitrogen $(Z=7)$, oxygen $(Z=8)$ and iron $(Z=26)$ for 15 November 2015-15 January 2016 (left) and 10 September 2017 (right) based on the ISO-15390 (dot-dashed lines) and BON 2014 (full lines) GCR models.

\section{Gale Crater Physical Environment}

Although dMEREM uses by default pre-processed data for the soil composition and density based on GRS data, in the present analysis the values used for the soil composition and density at Gale Crater are those measured by the Alpha-Particle X-ray Spectrometer (APXS) aboard the Curiosity Rover (Blake et al., 2013), displayed in Table 1, corresponding to a soil density of $2.8 \mathrm{~g} / \mathrm{cm}^{3}$ (Hassler et al., 2014). The atmospheric description corresponding to the epoch and season of September 2017 and for the Gale Crater location, as described by the European Mars Climate Data Base, was retrieved from the SPENVIS dMEREM interface corresponding to an atmospheric depth of $20 \mathrm{~g} / \mathrm{cm}^{2}$. The same description was used for the November 2015-January 2016 period dMEREM simulation, although the atmospheric density value retrieved for that epoch is $18.5 \mathrm{~g} / \mathrm{cm}^{2}$. This variation should be negligible for the proton flux predicted in the RAD measured energy domain. In a preliminary comparative study, the ratio between the AMEREM prediction for proton fluxes at the surface of Mars for atmospheric depths of $20 \mathrm{~g} / \mathrm{cm}^{2}$ and of $15 \mathrm{~g} / \mathrm{cm}^{2}$ was below $5 \%$ for detected proton energies below $100 \mathrm{MeV}$.

\section{Generation of Primary Particles}

The primary cosmic-ray fluxes used as inputs, on top of the Mars atmosphere, were obtained from two different propagation models: ISO-15930 model (Nymmik et al., 1992), as implemented on SPENVIS and from the Badhwar-O'Neill (BON) 2014 model (O’Neill, 2010; P M O'Neill TCS S Golge, 2015), as implemented on OLTARIS (Singleterry et al., 2011) web-based user interface for the corresponding periods of RAD data. For the period of early September 2017 a solar modulation parameter, $\Phi$, of 500 MV, obtained from the Oulu Neutron data base (Oulu Neutron Data Base, 2017), was used in OLTARIS in order to extract the expected BON2014 GCR spectra. Proton and ${ }^{4} \mathrm{He}$ GCR fluxes, as well as the fluxes of carbon, oxygen, nitrogen and iron nuclei for the two GCR models and for both analysed data periods are shown in Figure 1.

The differences between both GCR models are evident in the region below $\simeq 1 \mathrm{GeV} /$ nuc, where the spectra are affected by solar modulation. In this region the ISO-15390 model flux prediction is consistently below the $\mathrm{BON}-2014$ prediction, for all species and for both time periods, except for protons in the energy region between $\simeq 10 \mathrm{MeV}$ and $\simeq 1 \mathrm{GeV}$ for the 2017 period. This is due to the inherent differences between both models and also to the fact that the ISO-15390 model uses average yearly values for the sunspot number, on which the modulation parameter depends, whereas the BON2014 model uses monthly values for $\Phi$. In the region above $\simeq 1 \mathrm{GeV} /$ nuc, where GCR are no longer modulated by the solar activity, no differences between both models are observed.

Primary GCR fluxes were generated at the centre of the $500 \mathrm{~km} \times 500 \mathrm{~km}$ dMEREM pixel, from the top of the simulated atmosphere, at the altitude of $50 \mathrm{~km}$, according to a cosine-law distribution. For the analysis, GCR protons and ${ }^{4} \mathrm{He}$ nuclei were simulated in the energy interval from $12 \mathrm{MeV} /$ nuc to $0.9 \mathrm{TeV} /$ nuc, each sample consisting of $4.375 \times 10^{6}$ generated events. The heavier nuclei, ${ }^{12} \mathrm{C},{ }^{14} \mathrm{~N},{ }^{16} \mathrm{O}$, and ${ }^{56} \mathrm{Fe}$ were generated in the energy interval ranging from $12 \mathrm{MeV} /$ nuc to $0.5 \mathrm{TeV} /$ nuc, with $3.75 \times 10^{6}$ generated events in each data set. 


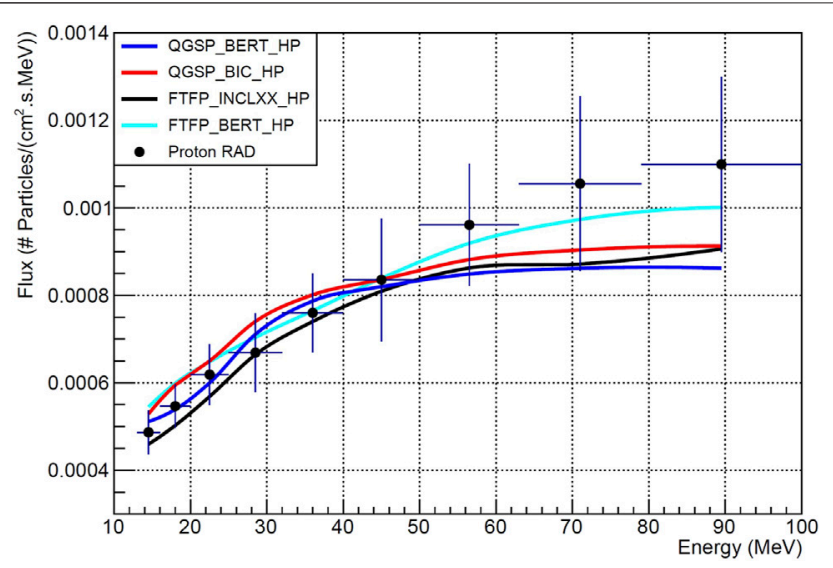

FIGURE 2 | Proton spectra reaching the Mars surface within the RAD field-of-view due to GCR-protons, helium, carbon and oxygen nuclei described with the GCR ISO-15390 model, simulated with dMEREM using four different physics lists compared to RAD proton differential flux measurements of September 2017.

\section{Geant4 Physics List Verification}

The first investigation of the dMEREM validation consisted in the verification of the most appropriate Geant 4 physics list for the present study. The Geant 4 toolkit allows to use a broad range of models for handling physical processes within different energy intervals. They are included in several reference physics lists which are routinely validated and updated with each release. In this study, dMEREM was tested with four different physics lists: QGSP_BIC_HP, QGSP_BERT_HP, FTFP_INCLXX_HP and FTFP_BERT_HP. ${ }^{2}$ These physics lists are named based on the combination of different hadronic models in different energy ranges, which are listed below, whereas standard electromagnetic (EM) processes are used by default in the four physics lists.

- High energy:

QGS-Quark Gluon String (QGS) model for high energy particles $(\geq 20 \mathrm{GeV})$;

FTF-Fritiof Model for string excitation and fragmentation for particles with energy $\geq 10 \mathrm{GeV}$;

- Below $10 \mathrm{GeV}$ :

BIC-Binary Cascade Model for primary protons and neutrons with energies $\leq 10 \mathrm{GeV}$.

BERT-Bertini Cascade Model for primary protons, neutrons, pions and kaons with energies $\leq 10 \mathrm{GeV}$;

- Intermediate energies:

P - Precompound model for de-excitation process for nucleon-induced reactions below $1-2 \mathrm{MeV}$;

INCL -Liege Intranuclear Cascade model (INCL++) for proton-, neutron- and pion-induced reactions below $3 \mathrm{GeV}$,

- Neutrons:

${ }^{2}$ http://geant4.web.cern.ch/geant4/support/proc_mod_catalog/physics_lists/ referencePL.shtml
HP-High Precision Neutron Model for neutrons with energies $\leq 20 \mathrm{GeV}$;

Both QGSP_BIC_HP and QGSP_BERT_HP models use the same Quark Gluon String model for the high energy range, while using different cascade models for energies $\leq 10 \mathrm{GeV}$, while FTFP_BERT_HP is recommended by the Geant 4 collaboration for cosmic-ray applications due to the most accurate treatment of very high energy particles. The FTFP_INCLXX_HP physics list was the one selected in the systematic study with the previous three physics lists presented in (Guo et al., 2019), which considered particles coming from primary protons and ${ }^{4} \mathrm{He}$ nuclei propagating in the Martian atmosphere, and therefore it is also considered in the present verification.

Sets of GCR protons and ${ }^{4} \mathrm{He}$ nuclei $\left(4.375 \times 10^{6}\right.$ generated particles per nuclei), and a lower statistics $\left(3.75 \times 10^{6}\right)$ of Oxygen and Carbon nuclei, with energies ranging from $12 \mathrm{MeV} /$ nuc to $0.5 \mathrm{TeV} /$ nuc were generated for each of the four chosen physics lists. The proton spectra predicted with dMEREM using the different physics lists originated from the generation of protons and helium, carbon and oxygen nuclei described with the ISO-15 390 model, integrated within the $36^{\circ}$ FOV RAD fieldof-view are compared with the RAD proton differential flux measurements of September 2017 (Ehresmann et al., 2018) in Figure 2.

Given the uncertainties associated to the measured RAD proton data set, the four tested physics lists yield compatible results for proton energies below $90 \mathrm{MeV}$. Therefore, in order to perform a deeper benchmark study for the Geant 4 physics lists choice, complementing what is reported in (Guo et al., 2019), comparisons of simulations of heavier GCR primaries - from Carbon to Iron-with $\mathrm{H}$ and $\mathrm{He}$ isotopes RAD measurements should be performed. Nevertheless, and for the purpose of this study, the FTFP_BERT_HP model was selected, since it displays a trend more compatible with the proton spectral data distribution for energies above $50 \mathrm{MeV}$.

\section{RESULTS}

dMEREM simulates the complete cascade of secondary particles produced both in the atmosphere and resulting from the interaction of the particles that arrive on the surface with the Mars soil, and all this information can be used to study the particle/radiation field on the surface of Mars or inside its atmosphere. Figure 3 displays the dMEREM predicted differential particle fluxes at the surface of Mars, at Gale Crater, after complete interaction with the Martian atmosphere and soil, for neutrons, photons, electrons, protons and alpha particles originated by GCR protons helium, carbon, oxygen, nitrogen and iron nuclei generated at the top of the Martian atmosphere, described with the ISO-15390 model for the September 2017 period. Albedo neutrons dominate the particle population at the surface of Mars for low energy values (below a few tens of keV) and there is also a gamma contribution, mostly albedo, between a few tens of $\mathrm{keV}$ and $1 \mathrm{MeV}$. The high energy part of the spectra is dominated by protons and ${ }^{4} \mathrm{He}$-only nuclei 

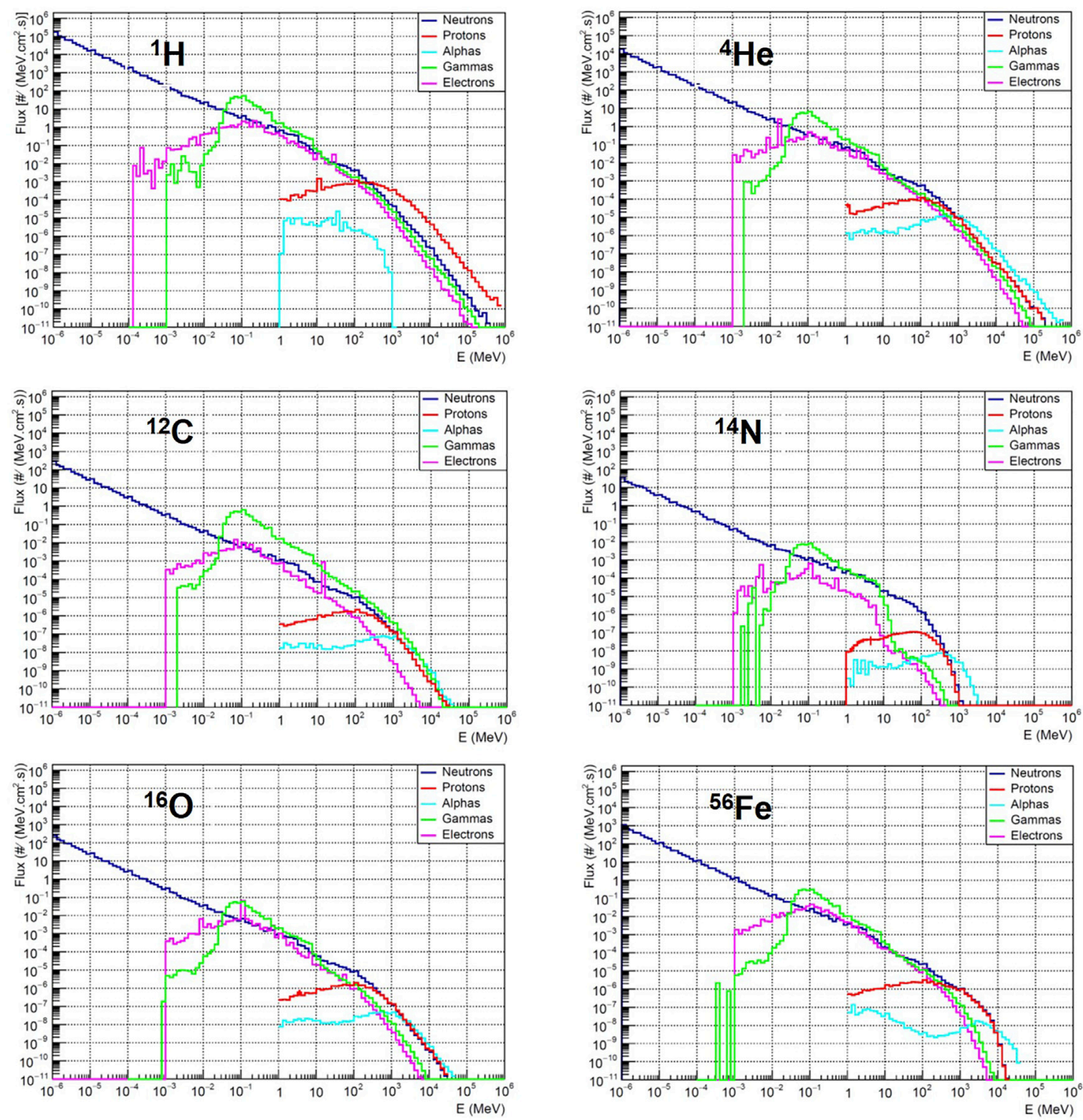

FIGURE 3 | Differential fluxes for a selected set of particle species predicted with dMEREM resulting from the different considered GCR sources - Protons, ${ }^{4}$ He, ${ }^{12} \mathrm{C},{ }^{14} \mathrm{~N},{ }^{16} \mathrm{O}$, and ${ }^{56} \mathrm{Fe}$ nuclei-with the ISO-15390 model for the conditions corresponding to early September 2017 at the Gale Crater.

with kinetic energy $\geq 1 \mathrm{MeV} /$ nuc were registered - and also by albedo neutrons. Neutrons produced in the interactions of energetic particles with the soil materials are a distinctive feature of Martian radiation spectra, with fluxes varying with the soil composition, in particular with the presence of water, which is an efficient absorber of energetic neutrons.

\section{Directionality Studies With dMEREM}

The angular distribution of secondary particles arriving at the Martian surface affects the calculation of the total particle flux, in particular when considering the Field-of-View of a detector such as RAD, which is limited in zenith angle to $36^{\circ}$ (WimmerSchweingruber et al., 2015). In order to investigate this effect, a directionality study was performed with protons originated from a combination of primary GCR-proton and ${ }^{4} \mathrm{He}$ ions based on the ISO-15390 model.

Figure 4 shows the zenith angle of protons (both upward and downward) with energies below $90 \mathrm{MeV}$, expected at the surface of Mars. The number of downward protons with zenith angles below $45^{\circ}$ is $\approx 13$ times larger than the number for those arriving from higher zenith angles. In fact, a fit to the total angular distribution of downward protons (blue data points) shows a depart from isotropy (red line in Figure 4), with $\chi^{2} / n d f=27.2$. However, the downward proton angular distribution approaches isotropy for zenith angles within the RAD field-of-view, with a fit value of $\chi^{2} / n d f=8$ for zenith angles below $36^{\circ}$ (black line in Figure 4). dMEREM results are thus compatible with the angular dependence of the radiation field on Mars measured with the 


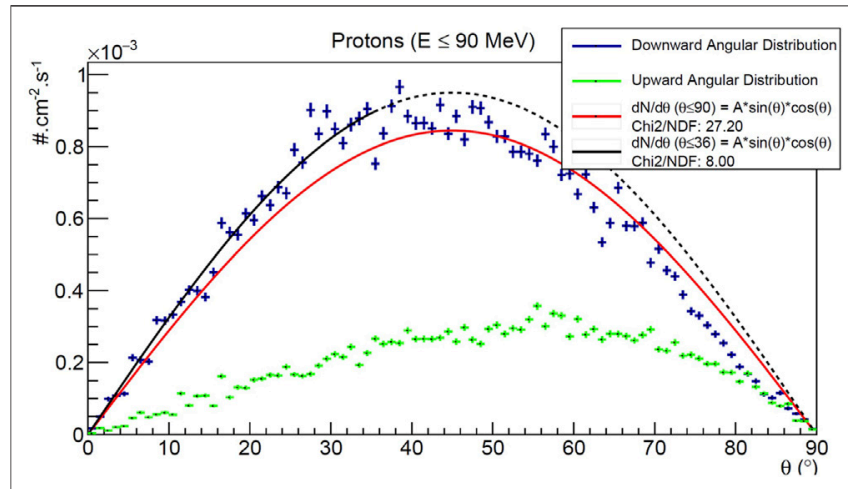

FIGURE 4 | Zenith angle distribution of protons with $\mathrm{E}<90 \mathrm{MeV}$ reaching the Mars surface, coming from primary spectra of GCR-protons and ${ }^{4} \mathrm{He}$. The zenith angle of both upward (green data points) and downward going protons (blue data points) is measured with respect to the corresponding normal vectors to the surface. The black and the red lines represent the fits to isotropy for the downward going protons within the fieldof-view of the RAD, and for all zenith angles, respectively.

RAD (Wimmer-Schweingruber et al., 2015), which was found to be close to isotropy within its field-of-view. The flux of upward going protons (green data points) is about $36 \%$ of the downward going component. It departs significantly from isotropy with $61.5 \%$ of the upward going protons coming from zenith angles above $45^{\circ}$ (with a larger horizontal component).

\section{dMEREM Prediction for RAD Measured Proton Spectra}

The differential proton fluxes expected at the Mars surface during the two selected periods of 15 November 2015 to 15 January 2016 and early September 2017, were estimated with dMEREM and compared with the RAD measurements for those periods, presented in (Ehresmann et al., 2017) and (Ehresmann et al., 2018), respectively.
Figures 5, 6 display the comparison of the measured RAD proton spectra for proton energies below $100 \mathrm{MeV}$ for the two periods considered, compared to the corresponding dMEREM predictions (blue lines) for the ISO-15 390 (left) and BON 2014 (right) GCR models, including the breakdown of the dMEREM flux predictions into primary (red lines) and secondary (cyan lines) components. As expected, in this low energy regime, the major component of protons arriving to the soil are of secondary origin, coming from interactions in the atmosphere and also with the Martian soil.

If a $\chi^{2}$ statistical test is performed for the 2015-2016 period, the BON2014 dMEREM prediction yields a value of $\chi^{2}=1.54$, compared to $\chi^{2}=4.67$ for the ISO-15390 model, for 8 degrees of freedom. For the 2017 period the $\chi^{2}$ test for the BON2014 prediction yields a value of $\chi^{2}=4.65$, compared to $\chi^{2}=3.33$ for the ISO-15390 prediction, for 7 degrees of freedom. Therefore, the results obtained with dMEREM with both GCR models - ISO-15390 and BON2014-are found to be compatible with the proton RAD measurements for both periods. Nevertheless it is observed that the measured proton spectra display a trend towards a steeper increase with energy, when compared to the predictions of both models.

Figure 7 and Table 2 display ISO-15390 model based dMEREM predictions for the conditions of November 2015January 2016. In Figure 7 dMEREM predictions for the proton differential fluxes broken down by their origin in terms of different primary GCR species- $\mathrm{H}, \mathrm{He}, \mathrm{C}, \mathrm{N}, \mathrm{O}$ and Fe nuclei are compared with the corresponding RAD data. In Table 2 the integral fluxes of protons with energies ranging from 1 to $90 \mathrm{MeV}$ at the Gale Crater are displayed for the contributions from each primary GCR species, as well as the corresponding interplanetary integral fluxes, for primary energies ranging from $1 \mathrm{MeV} /$ nuc to $500 \mathrm{GeV} /$ nuc. It can be observed that the contribution of Helium4 primaries to the total number of detected protons at the surface is of the order of $10 \%$, whereas the contributions from Iron, Carbon and Oxygen nuclei are of at most $0.2 \%$ each, and the contribution from Nitrogen is one order of magnitude smaller.
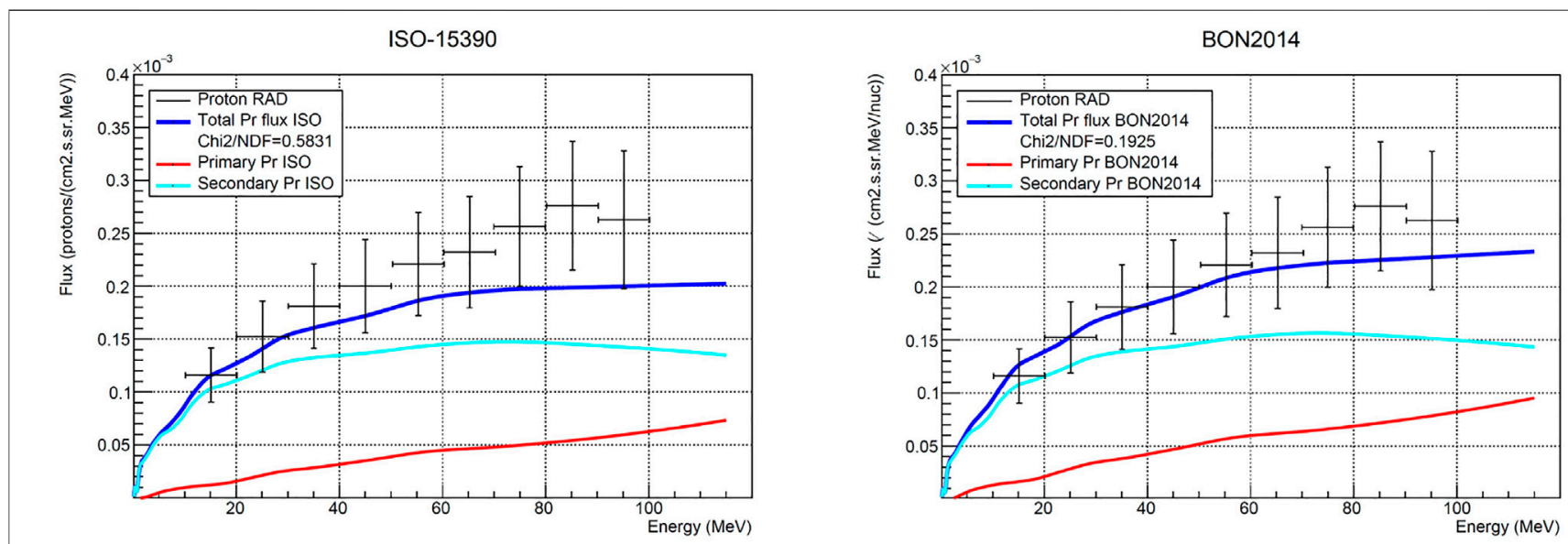

FIGURE 5 | Differential proton flux spectra obtained with dMEREM compared to GCR-induced proton spectrum measured by RAD in November 2015-January 2016, extracted from (Ehresmann et al., 2017). Protons from primary (red) and secondary (cyan) origin are shown. dMEREM prediction based on ISO-15390 flux prediction is shown on the left, while the prediction based on BON2014 model is shown on the right. 

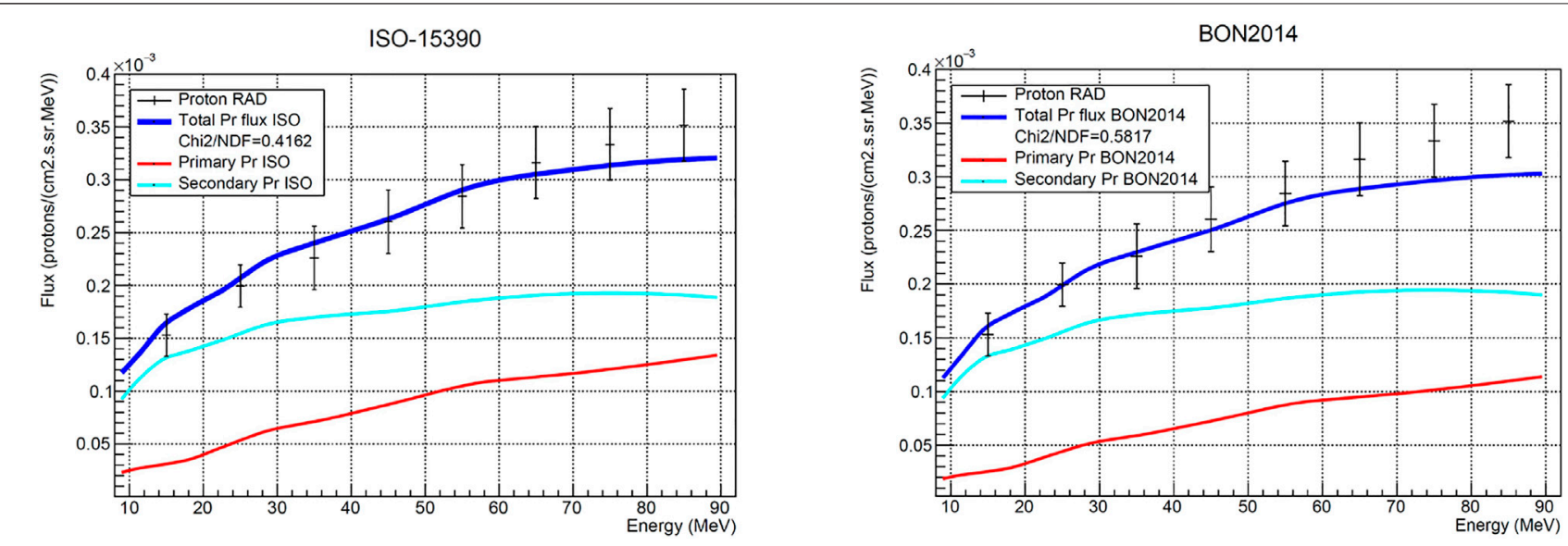

FIGURE 6 | Differential proton flux spectra obtained with dMEREM compared to GCR-induced proton spectrum measured by RAD in September 2017, extracted from (Ehresmann et al., 2018). Protons from primary (red) and secondary (cyan) origin are shown. dMEREM prediction based on ISO-15390 model (left) and on BON2014 model (right) are shown.

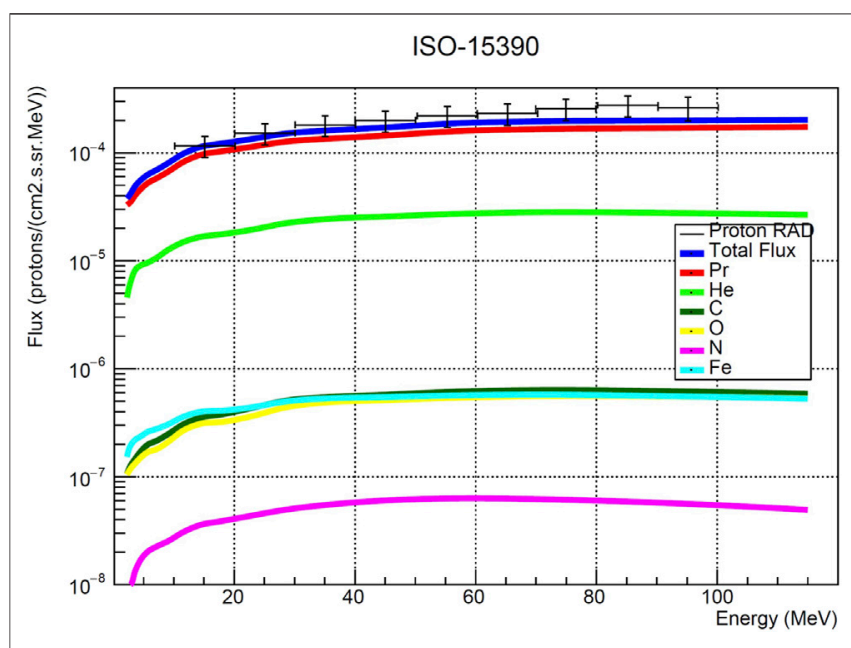

FIGURE 7 | Differential proton flux spectra obtained with dMEREM compared to GCR-induced proton spectrum measured by RAD in 15 November 2015-15 January 2016 (Ehresmann et al., 2017). Proton fluxes induced by primary $\mathrm{H}, \mathrm{He}, \mathrm{C}, \mathrm{N}, \mathrm{O}$, and Fe nuclei are based on ISO15390 model.

\section{DISCUSSION}

dMEREM is a Geant 4 based model for the detailed description of the energetic radiation environment in Mars, developed under the ESA's MarsREM Project. It is an end-to end model, based on a Geant4 Monte-Carlo core, interfaced with Martian atmospheric circulation models, soil composition data bases and with a Martian crustal magnetic field model. It performs detailed simulations of the propagation of energetic particles at locations in Mars and on its Moons, namely in the Martian orbit, inside its atmosphere, at the Martian surface or underground. The first release of dMEREM was interfaced with SPENVIS 2009 (Heynderickx et al., 2004; Kruglanski et al., 2009), where it is accessible to the community. It uses a very detailed description of the Mars atmosphere, extracting data from the Mars Climate DataBase Model or MarsGRAM, and soil composition data from the GRS aboard the Mars Odyssey. For the Mars atmosphere choice, the model uses epoch, solar longitude, day/night conditions, location (latitude-longitude) and altitude dependent profiles. It also allows different soil composition data sets to be simulated depending on the user choice. This possibility is available in the dMEREM SPENVIS implementation.

Given the flexibility both of the dMEREM core $\mathrm{C}++$ implementation and of its python based interfaces, the framework can be adapted to new data characterising the Martian soil and atmosphere coming from present and future missions of NASA and ESA exploration programmes. dMEREM is therefore a powerful tool for making forecasts of the Martian ionising radiation environment at chosen locations on Mars and for different epochs, and it can be used for benchmarking of other models, in particular, those relying on transfer functions and ray tracing techniques.

dMEREM was validated in the past through comparison with available simulation codes like HZTERN, since no Mars Radiation data existed at the time (McKenna-Lawlor et al., 2012b). In the present work GCR-induced proton differential spectra, registered by the RAD in the period from November 2015 to January 2016 and in the first days of September 2017, were used in the validation of dMEREM.

The dMEREM characterisation of the Martian ionising radiation environment relies on different models for the propagation of GCR in the Heliosphere and on data and models for describing the physical environment quantities affecting particle propagation in the planetary atmosphere and surface. Two different GCR models were used in the validation, the ISO-15 390 (Nymmik et al., 1992) and on the BadhwarO’Neill 2014 (P M O’Neill TCS S Golge, 2015) model. dMEREM was input with the European Mars Climate Data Base (Forget et al., 1999; Millour et al., 2018) atmospheric information corresponding to the analysed time periods and a soil composition as measured by the APXS instrument (Blake et al., 2013) aboard Curiosity. 
TABLE 2 | Secondary protons integral fluxes predicted with dMEREM for Gale Crater, for two energy domains - the 10-90 MeV interval corresponding to the RAD measurements - for the conditions of the period from November 2015 to 15/01/2,016 and based on the ISO-15390 model. The proton fluxes at the surface and their relative abundances are shown for the primary GCR species originating them. The corresponding integral fluxes of primary particles are also shown. Uncertainties are statistical only.

\begin{tabular}{|c|c|c|c|c|c|}
\hline $\begin{array}{l}\text { Primary GCR } \\
\text { species }\end{array}$ & $\begin{array}{c}\text { Secondary proton } \\
\text { flux } 10-90 \mathrm{MeV} \\
{\left[10^{-3} /\left(\mathrm{cm}^{2} . \text { sr.s }\right)\right]}\end{array}$ & Relative contribution \% & $\begin{array}{l}\text { Secondary proton } \\
\text { flux } 10-500 \mathrm{MeV} \\
{\left[10^{-3} /\left(\mathrm{cm}^{2} . \mathrm{sr} . \mathrm{s}\right)\right]}\end{array}$ & Relative contribution \% & $\begin{array}{c}\text { GCR flux } \\
1 \mathrm{MeV} / \text { nuc }< \\
\mathrm{E}< \\
500 \mathrm{GeV} / \text { nuc }\left[10^{-3} /\left(\mathrm{cm}^{2} \text {.sr.s }\right)\right]\end{array}$ \\
\hline Protons & $1.92 \pm 0.01$ & $89.7 \pm 0.6$ & $5.34 \pm 0.02$ & $92.4 \pm 0.5$ & $215 \pm 1$ \\
\hline Helium & $0.213 \pm 0.001$ & $9.96 \pm 0.06$ & $0.42 \pm 0.02$ & $7.33 \pm 0.05$ & $23.4 \pm 0.2$ \\
\hline Carbon & $(4.21 \pm 0.03) \cdot 10^{-3}$ & $0.197 \pm 0.002$ & $(7.81 \pm 0.07) \cdot 10^{-3}$ & $0.135 \pm 0.001$ & $0.655 \pm 0.001$ \\
\hline Nitrogen & $(4.78 \pm 0.07) \cdot 10^{-4}$ & $0.0224 \pm 0.0003$ & $(7.6 \pm 0.1) \cdot 10^{-4}$ & $0.0131 \pm 0.0002$ & $0.611 \pm 0.005$ \\
\hline Oxygen & $(3.42 \pm 0.02) \cdot 10^{-3}$ & $0.016 \pm 0.001$ & $(6.57 \pm 0.05) \cdot 10^{-3}$ & $0.114 \pm 0.001$ & $0.173 \pm 0.001$ \\
\hline Iron & $(2.663 \pm 0.009) \cdot 10^{-3}$ & $0.0125 \pm 0.0006$ & $(5.99 \pm 0.02) \cdot 10^{-3}$ & $0.104 \pm 0.0005$ & $0.0691 \pm 0.0003$ \\
\hline Total & $2.14 \pm 0.01$ & & $5.77 \pm 0.02$ & & $239.9 \pm 1.0$ \\
\hline
\end{tabular}

The sum of the contributions of all generated GCR primaries to the: secondary integral proton flux within 10 to 90 MeV (1st column); secondary integral proton flux within 10 to $500 \mathrm{GeV}$ (2nd collumn); Primary GCR integral flux within the energy range 1-500GeV/nuc (last column).

In the validation process, the accuracy of dMEREM in the simulation of secondary proton spectra at the Martian surface due primary GCR species comprising protons, helium, carbon and oxygen nuclei, was verified using four different Geant 4 physics lists. Among these, the FTFP_BERT_HP physics list was then used in the validation of dMEREM although all the physics lists tested were in reasonable agreement with data for the proton energy range below $50 \mathrm{MeV}$. Above this energy, between 50 and $100 \mathrm{MeV}$, FTFP_BERT_HP showed a better agreement with the RAD stopping proton measurements. A similar study (Guo et al., 2019) of the same four physics lists selected FTFP_INCLXX_HP physics list as the more appropriate, using GCR proton and ${ }^{4} \mathrm{He}$ as primaries, based on the agreement with RAD data for several $\mathrm{H}$ and $\mathrm{He}$ isotopes. In the present study, in addition to GCR protons and ${ }^{4} \mathrm{He}$ primaries, ${ }^{12} \mathrm{C}$ and ${ }^{16} \mathrm{O}$ nuclei were used. Although there are other secondaries such as electrons, positrons, muons that also contribute to the Mars radiation environment that could be used to further compare the different physics lists results, these were not used as additional information in either study.

Directionality studies of the proton spectrum at the Martian surface performed with dMEREM (using the ISO-15390 model, for the 2017 data set conditions) showed that the global zenith angle distribution of secondary protons at the Martian surface does not correspond to an isotropic distribution, in particular the proton albedo from the interaction of cosmic rays with the Martian soil is expected to be highly anisotropic. However, it was shown that for downward going protons, dMEREM predictions for the fluxes of protons with energies below $\approx 100 \mathrm{MeV}$ are close to isotropy within the RAD field-of-view.

dMEREM predictions for the proton fluxes at the Martian surface originated in the interactions of primary GCR nuclei in the Martian Atmosphere and soil for the two analysed periods-from November 2015 to January 2016 and for early September 2017-based on the ISO-15 390 and on the BadhwarO'Neill 2014 models, were found to be consistent with the stopping proton data measured by $\mathrm{RAD}$ during those periods. This is confirmed from the $\chi^{2}$ goodness of fit tests performed to compare dMEREM predictions and data, with the best fit corresponding to the dMEREM prediction based on the BON2014 model for the November 2015-January 2016 data.

It should however be noted that in this work the shielding and scattering effect of the Curiosity rover materials, and of the RAD detector itself, are not being simulated, and therefore any additional effect due to the production of secondary particles is not included. Additionally, in this study the surface of Mars is considered to be flat and any effect due to topography is also not being taken into account. Future work will explore these systematic effects, as well as the dMEREM predictions for the RAD measurements for other secondary particle species, and for the radiation environment on the Martian surface during SEP events.

\section{DATA AVAILABILITY STATEMENT}

The raw data supporting the conclusion of this article will be made available by the authors, without undue reservation.

\section{AUTHOR CONTRIBUTIONS}

GP is accountable for all aspects of the work, having ensured that questions related to the accuracy or integrity of any part of the work were appropriately investigated. She coordinated the analysis and interpretation of the results and she is the redactor of the submitted version of the work, having revised it critically for important intellectual content and having provided approval for publication of the content. LA performed the Geant 4 physics lists benchmarking analysis. She produced significant contributions to the conception of the work, namely in the production of all simulation data sets and in the preparation of the validation analysis. She contributed to the data analysis and to its interpretation. She produced the first draft of the work. MP was responsible for the analysis and interpretation of dMEREM directional data, including the verification of the correct use of the GCR models for the analysed data periods. He participated in the critical revision of the work for important intellectual content. 


\section{REFERENCES}

Adams ea, J. H. (1981). Cosmic Ray Effects on Microelectronics. Part I, the Near Earth Particle Environment. Washington, U.S.A: NRL MemoRep. 4506 Pt.I. Publ. Naval Research Laboratory.

Agostinelli, S., Allison, J., Amako, K., Apostolakis, J., Araujo, H., Arce, P., et al. (2003). Geant4-a Simulation Toolkit. Nucl. Instr. Methods Phys. Res. Section A: Acc. Spectrometers, Detectors Associated Equipment 506, 250-303. doi:10.1016/ s0168-9002(03)01368-8

Allison, J., Amako, K., Apostolakis, J., Arce, P., Asai, M., Aso, T., et al. (2016). Recent Developments in Geant4. Nucl. Instr. Methods Phys. Res. Section A: Acc. Spectrometers, Detectors Associated Equipment 835, 186-225. doi:10.1016/j. nima.2016.06.125

Allison, J., Amako, K., Apostolakis, J., Araujo, H., Arce Dubois, P., Asai, M., et al. (2006). Geant4 Developments and Applications. IEEE Trans. Nucl. Sci. 53, 270-278. doi:10.1109/tns.2006.869826

Baumstark-Khan, C., and Facius, R. (2001). "Life under Conditions of Ionizing Radiation Astrobiology," in The Quest for the Conditions of Life (Berlin, Germany: Springer-Verlag).

Blake, D. F., Morris, R. V., Kocurek, G., Morrison, S. M., Downs, R. T., Bish, D., et al. (2013). Curiosity at Gale Crater, Mars: Characterization and Analysis of the Rocknest Sand Shadow. Science 341, 1239505. doi:10.1126/science.1239505

Böhlen, T. T., Cerutti, F., Chin, M. W., Fassò, A., Ferrari, A., Ortega, P. G., et al. (2014). The FLUKA Code: Developments and Challenges for High Energy and Medical Applications. Nucl. Data Sheets 120, 211-214.

Boynton, W. V., Feldman, W. C., Mitrofanov, I. G., Evans, L. G., Reedy, R. C., Squyres, S. W., et al. (2004). The mars Odyssey Gamma-ray Spectrometer Instrument Suite. Space Sci. Rev. 110, 37-83. doi:10.1023/B:SPAC.0000021007. 76126.15

Cabrol, N. A., Grin, E. A., Newsom, H. E., Landheim, R., and McKay, C. P. (1999). Hydrogeologic Evolution of Gale Crater and its Relevance to the Exobiological Exploration of Mars. Icarus 139, 235-245. doi:10.1006/icar.1999.6099

Cucinotta, F., Hu, S., Schwadron, N., Kozarev, K., Townsend, L., and Kim, M. (2010). Space Radiation Risk Limits and Earth Moon Mars Environmental Models. Space Weather 8, 9. doi:10.1029/2010sw000572

Cucinotta, F. (2012). Space Radiation Cancer Risk Projections and Uncertainties. Tech. Rep. (Nasa/tp-2013-217375).

Da Pieve, F., Gronoff, G., Guo, J., Mertens, C. J., Neary, L., Gu, B., et al. (2021). Radiation Environment and Doses on Mars at Oxia Planum and Mawrth Vallis: Support for Exploration at Sites with High Biosignature Preservation Potential. J. Geophys. Res. Planets 126, e2020JE006488. doi:10.1029/2020je006488

[Dataset] Oulu Neutron Data Base (2017). Oulu Neutron Data Base. Available at: https://cosmicrays.oulu.fi/phi/phi_table_2017.txt.

De Angelis, G., Badavi, F. F., Blattnig, S. R., Clowdsley, M. S., Nealy, J. E., Qualls, G. D., et al. (2007). Modeling of the Martian Environment for Radiation Analysis. Nucl. Phys. B - Proc. Supplements 166, 184-202. doi:10.1016/j.nuclphysbps. 2006.12.035

de Wet, W. C., and Townsend, L. W. (2017). A Calculation of the Radiation Environment on the Martian Surface. Life Sci. Space Res. 14, 51-56. doi:10. 1016/j.lssr.2017.07.008

Durante, M., and Cucinotta, F. A. (2008). Heavy Ion Carcinogenesis and Human Space Exploration. Nat. Rev. Cancer 8, 465-472. doi:10.1038/nrc2391

Ehresmann, B., Hassler, D. M., Zeitlin, C., Guo, J., Wimmer-Schweingruber, R. F., Matthiä, D., et al. (2018). Energetic Particle Radiation Environment Observed by RAD on the Surface of Mars during the September 2017 Event. Geophys. Res. Lett. 45, 5305-5311. doi:10.1029/2018gl077801

Ehresmann, B., Zeitlin, C. J., Hassler, D. M., Matthiä, D., Guo, J., WimmerSchweingruber, R. F., et al. (2017). The Charged Particle Radiation Environment on Mars Measured by MSL/RAD from November 15, 2015 to January 15, 2016. Life Sci. Space Res. 14, 3-11. doi:10.1016/j.lssr.2017.07.004

[Dataset] Ferrari, A., Sala, P. R., Fasso, A., and Ranft, J. (2005). FLUKA: A MultiParticle Transport Code.

Flores-McLaughlin, J. (2017). Radiation Transport Simulation of the Martian GCR Surface Flux and Dose Estimation Using Spherical Geometry in PHITS Compared to MSL-RAD Measurements. Life Sci. Space Res. 14, 36-42. doi:10.1016/j.lssr.2017.07.007
Forget, F., Hourdin, F., Fournier, R., Hourdin, C., Talagrand, O., Collins, M., et al. (1999). Improved General Circulation Models of the Martian Atmosphere from the Surface to above $80 \mathrm{Km}$. J. Geophys. Res. 104, 24155-24175. doi:10.1029/ 1999JE001025

Gonçalves, P., Keating, A., Valente, S., Truscott, P., Lei, F., Desorgher, L., et al. (2009). "MarsREM: The Mars Energetic Radiation Environment Models," in 31st International Cosmic Ray Conference, ICRC 2009.

Grotzinger, J. P., Crisp, J., Vasavada, A. R., Anderson, R. C., Baker, C. J., Barry, R., et al. (2012). Mars Science Laboratory Mission and Science Investigation. Space Sci. Rev. 170, 5-56. doi:10.1007/s11214-012-9892-2

Guo, J., Banjac, S., Röstel, L., Terasa, J. C., Herbst, K., Heber, B., et al. (2019). Implementation and Validation of the GEANT4/AtRIS Code to Model the Radiation Environment at Mars. J. Space Weather Space Clim. 9, A2. doi:10. $1051 /$ swsc/2018051

Guo, J., Zeitlin, C., Wimmer-Schweingruber, R. F., McDole, T., Kühl, P., Appel, J. C., et al. (2018). A Generalized Approach to Model the Spectra and Radiation Dose Rate of Solar Particle Events on the Surface of Mars. Astronomical J. 155 49. doi:10.3847/1538-3881/aaa085

Hassler, D. M., Zeitlin, C., Wimmer-Schweingruber, R. F., Ehresmann, B., Rafkin, S., Eigenbrode, J. L., et al. (2014). Mars' Surface Radiation Environment Measured with the Mars Science Laboratory's Curiosity Rover. Science 343, 1244797. doi:10.1126/science. 1244797

Hassler, D. M., Norbury, J. W., and Reitz, G. (2017). Mars Science Laboratory Radiation Assessment Detector (MSL/RAD) Modeling Workshop Proceedings. Life Sci. Space Res. 14, 1-2. doi:10.1016/j.lssr.2017.06.004

Hassler, D. M., Zeitlin, C., Wimmer-Schweingruber, R. F., Böttcher, S., Martin, C., Andrews, J., et al. (2012). The Radiation Assessment Detector (RAD) Investigation. Space Sci. Rev. 170, 503-558. doi:10.1007/s11214-012-9913-1

Heynderickx, D., Quaghebeur, B., Wera, J., Daly, E., and Evans, H. (2004). New Radiation Environment and Effect Models in Esa's Space Environment Information System (SPENVIS). Space Weather 2, S10s03. doi:10.1029/ 2004sw000073

Justus, C. G., James, B. F., Bougher, S. W., Bridger, A. F. C., Haberle, R. M., Murphy, J. R., et al. (2002). Mars-GRAM 2000: A Mars Atmospheric Model for Engineering Applications. Adv. Space Res. 29, 193-202. doi:10.1016/s02731177(01)00569-5

Kruglanski, M., Messios, N., De Donder, E., Gamby, E., Calders, S., Hetey, L., et al. (2009). "Last Upgrades and Development of the Space Environment Information System (SPENVIS)," in Proceedings of the European Conference on radiation and its effects on components and systems, RADECS, 563-565. doi:10.1109/radecs.2009.5994715

Matthiä, D., and Berger, T. (2017). The Radiation Environment on the Surface of Mars - Numerical Calculations of the Galactic Component with GEANT4/ PLANETOCOSMICS. Life Sci. Space Res. 14, 57-63. doi:10.1016/j.lssr.2017. 03.005

McKenna-Lawlor, S., Gonçalves, P., Keating, A., Morgado, B., Heynderickx, D., Nieminen, P., et al. (2012). Characterization of the Particle Radiation Environment at Three Potential landing Sites on Mars Using ESA's MEREM Models. Icarus 218, 723-734. doi:10.1016/j.icarus.2011.04.004

McKenna-Lawlor, S., Gonçalves, P., Keating, A., Reitz, G., and Matthiä, D. (2012). Overview of Energetic Particle Hazards during Prospective Manned Missions to mars. Planet. Space Sci. 63-64, 123-132. doi:10.1016/j.pss.2011.06.017

Millour, E., Forget, F., Spiga, A., Vals, M., Zakharov, V., Montabone, L., et al. (2018). “The Mars Climate Database (Version 5.3)," in Scientific Workshop: "From Mars Express to ExoMars", ESAC Madrid, Spain, 27-28 February 2018. doi:10.1029/1999JE001025

Nymmik, R. A., Panasyuk, M. I., Pervaja, T. I., and Suslov, A. A. (1992). A Model of Galactic Cosmic ray Fluxes. Int. J. Radiat. Appl. Instrumentation. D. Nucl. Tracks Radiat. Measurements 20, 427-429. doi:10.1016/1359-0189(92)90028-t

O’Neill, P. M. (2010). Badhwar-O’Neill 2010 Galactic Cosmic Ray Flux Model-Revised. IEEE Trans. Nucl. Sci. 57, 3148-3153.

P M O’Neill Tcs S Golge (2015). Badhwar - O'Neill 2014 Galactic Cosmic Ray Flux Model Description. Houston, Texas: NASA.

Pelliccioni, M. (2000). Overview of Fluence-To-Effective Dose and Fluence-ToAmbient Dose Equivalent Conversion Coefficients for High Energy Radiation Calculated Using the FLUKA Code. Radiat. Prot. Dosimetry 88, 279-297. doi:10.1093/oxfordjournals.rpd.a033046 
Ratliff, H. N., Smith, M. B. R., and Heilbronn, L. (2017). Simulation of the GCR Spectrum in the Mars Curiosity Rover's RAD Detector Using MCNP6. Life Sci. Space Res. 14, 43-50. doi:10.1016/j.lssr.2017.07.003

Sato, T., Niita, K., Matsuda, N., Hashimoto, S., Iwamoto, Y., Noda, S., et al. (2013). Particle and Heavy Ion Transport Code System, PHITS, Version 2.52. J. Nucl. Sci. Technol. 50, 913-923. doi:10.1080/00223131.2013.814553

Simpson, J. A. (1983). Elemental and Isotopic Composition of the Galactic Cosmic Rays. Annu. Rev. Nucl. Part. Sci. 33, 323-382. doi:10.1146/annurev.ns.33.120183.001543

Singleterry, R. C., Blattnig, S. R., Clowdsley, M. S., Qualls, G. D., Sandridge, C. A., Simonsen, L. C., et al. (2011). OLTARIS: On-Line Tool for the Assessment of Radiation in Space. Acta Astronautica 68, 1086-1097. doi:10.1016/j.actaastro.2010.09.022

Slaba, T. C., and Stoffle, N. N. (2017). Evaluation of HZETRN on the Martian Surface: Sensitivity Tests and Model Results. Life Sci. Space Res. 14, 29-35. doi:10.1016/j.lssr.2017.03.001

Slaba, T. C., Wilson, J. W., Badavi, F. F., Reddell, B. D., and Bahadori, A. A. (2016). Solar Proton Exposure of an ICRU Sphere within a Complex Structure Part II: Ray-trace Geometry. Life Sci. Space Res. 9, 77-83. doi:10.1016/j.lssr.2016.05.001

Wimmer-Schweingruber, R. F., Köhler, J., Hassler, D. M., Guo, J., Appel, J.-K., Zeitlin, C., et al. (2015). On Determining the Zenith Angle Dependence of the
Martian Radiation Environment at Gale Crater Altitudes. Geophys. Res. Lett. 42, 557. doi:10.1002/2015GL066664

Conflict of Interest: The authors declare that the research was conducted in the absence of any commercial or financial relationships that could be construed as a potential conflict of interest.

Publisher's Note: All claims expressed in this article are solely those of the authors and do not necessarily represent those of their affiliated organizations, or those of the publisher, the editors and the reviewers. Any product that may be evaluated in this article, or claim that may be made by its manufacturer, is not guaranteed or endorsed by the publisher.

Copyright (C) 2022 Gonçalves, Arruda and Pinto. This is an open-access article distributed under the terms of the Creative Commons Attribution License (CC BY). The use, distribution or reproduction in other forums is permitted, provided the original author(s) and the copyright owner(s) are credited and that the original publication in this journal is cited, in accordance with accepted academic practice. No use, distribution or reproduction is permitted which does not comply with these terms. 